\title{
Variabilité polyphénolique et systématique du pin sylvestre Pinus sylvestris L
}

\author{
P Lebreton ${ }^{*}$ C Laracine-Pittet, C Bayet, J Lauranson \\ Laboratoire de biochimie végétale de l'université Lyon-l 69622 Villeurbanne cedex France \\ et GRECO CNRS 13043, \\ Écologie des forêts méditerranéennes
}

(Reçu le $1^{\text {er }}$ décembre 1988 ; accepté le 13 juin 1989)

\begin{abstract}
Résumé - Le Pin sylvestre est sujet à polymorphisme polyphénolique tranché : chez un premier chimiotype, la prodelphinidine constitue le flavonoïde majeur; chez le second, la voie de la phényl-trihydroxylation est réprimée au profit de la dihydroxylation : la procyanidine et la quercétine augmentent, tandis qu'apparaît la dihydroquercétine (= taxifoline, T). Le premier chimiotype (ou "chimiomorphe"), dit T-, est quasiment seul présent chez les populations “montagnardes" ou "froides" de l'espèce, de la Laponie à la Sierra Nevada, en passant par le Jura, les Alpes, le Massif central et les Pyrénees ; un cas extrême est offert par les individus écossais (procyanidine et quercétine vestigiales), relevant de la var scotica Schott. Le second chimiotype, $T+$, devient notable, voire prédominant, chez les populations planitiaires et "chaudes" ; il représente ainsi 29 à $62 \%$ des individus en Suedde centrale et méridionale, $43 \%$ dans les basses Vosges, $32 \%$ dans le Vaucluse (France), $35 \%$ en Europe de l'Est. Du point de vue de la biogenèse chimique, ce second type est plus divers, et plus évolué. Outre la reconnaissance de la variété d'Ecosse, il est donc suggéré l'existence de 2 groupes infraspécifiques chez Pinus sylvestris $L$ : un groupe archaïque boréo-alpin " $A$ " (comme altitude) ayant persisté dans les zones-refuges élevées lors des dernières périodes interglaciaires ; un groupe plus récent et plus mobile "P" (comme plaine) ayant (re)colonisé (depuis le Nord-Est ?) les zones basses à la faveur des périodes de refroidissement. Cette proposition n'est pas contradictoire avec des hypotheses antérieures formulées par Guinier. II n'est pas exclu que la reconnaissance et la sélection de ces chimiotypes flavoniques puisse constituer une aide à la pratique des reboisements, ou à leur analyse a posteriori.
\end{abstract}

Pin sylvestre / conifères / polyphénols / polymorphisme / caractérisation infraspécifique

Summary - Polyphenol variability and systematics of the Scots Pine (Pinus sylvestris $\mathbf{L}$ ). Within the Scots Pine, we observed a marked polyphenolic polymorphism: in the first chemomorph, prodelphinidin constitutes the main flavonoid whereas phenyl-trihydroxylation is suppressed by dihydroxylation in the second chemomorph : procyanidin and quercetin increase; at the same time dihydroquercetin (= taxifolin, $T$ ) appears. The first morph, called $T$-, is largely predominant within the "mountain" or "cold" populations of Scots Pine, from

* Correspondance et tirés à part 
Lapland to Sierra Nevada (Spain), via the Jura, Alps, Massif central and Pyrenees: an extreme case is represented by the Scottish specimens (procyanidin and quercetin become vestigial) related to the var scotica Schott. The second morph. T+, becomes numerous and even predominant within the "plain" and "hot" populations. This morph constitutes $29-62 \%$ of individuals in Central and Southern Sweden, $43 \%$ in Basses-Vosges, 32\% in Vaucluse (France), $35 \%$ in Eastern Europe. From a biochemical point of view, the second morph is more diversified and highly evolved. In addition to the distinction of the Scottish variety, we propose thus to recognize 2 other subspecific taxa within Pinus sylvestris $L: 1$ archaic and boreo-alpine group called "A" (as for Altitude), which has remained in refuge zones at high elevations during the interglacial periods; another group, more recent and more mobile, called " $P$ " (as for Plain), which has (re)colonised the lowlands from the North-East during the cold periods. This proposition is not contrary to the former hypothesis expressed by Guinier. It would not be unreasonable to state that the determination and the selection of these flavonic chemomorphs could help forestry, or its critical analysis a posteriori.

Scots Pine / conifers / polyphenols / polymorphism / infraspecific characterization

\section{INTRODUCTION}

«De tous les pins, le Pin sylvestre est celui qui à l'aire naturelle la plus vaste: du Portugal à la mer d'Okhostsk, soit $9000 \mathrm{~km}$ environ d'ouest en est ; du nord de la Norvège au sud de l'Espagne, soit $4000 \mathrm{~km}$ du nord au sud ; en altitude, l'espece se rencontre depuis le niveau de la mer jusqu'à l'étage montagnard, ou même subalpin si la pluviosité est assez faible" (Arbez, 1987).

Dans de telles conditions, rien de très étonnant à constater chez le Pin sylvestre une grande variabilité morphologique et physiologique (port, rusticité, gélivité, productivité, etc), expliquant pour une bonne part les divergences et l'embarras des systématiciens. Espérant peut-être rendre compte de la réalité de terrain, Carslisle (1958, cité par Gaussen, 1960) va jusqu'à inventorier pas moins de 144 "types» distincts ; plus modestes, et plus réalistes, Jalas et Suominen (1973) cartographient l'espèce «as a collective taxon only" dans l'Atlas Florae Europeae.

Gaussen, quant à lui, propose de reconnaître 7 sous-ensembles, dont cer- tains correspondent à des sous- espèces ou variétés classiquement reconnues, notamment par des forestiers : le Pin de Riga (var rigensis Loud), le Pin d'Ecosse (var scotica Schott), le Pin des Vosges (var haguenensis Loud, rapprochée il est vrai des var bororussica Schott, engadinensis Heer, nevadensis Christ, etc, comme groupe des basses altitudes de l'aire moyenne et méridionale). De Ferre (1979) va dans le même sens et après une étude biométrique des cotylédons et des plantules - définit 2 grands ensembles, de part et d'autre du $46^{\mathrm{e}}$ parallèle : un groupe septentrional comprenant 3 sous-groupes (scandinave, écossais, centre-européen), un groupe méridional opposant un sousgroupe oriental à une vaste entité couvrant les Alpes et toute l'Espagne.

Devant une situation aussi complexe, voire confuse, il nous a semblé intéressant et utile d'appliquer à Pinus sylvestris la démarche chimiotaxinomique, fondée sur l'enchaînement causal ADN nucléaire/ARN messager/protéines enzymatiques/substrats primaires/métabolites secondaires ; en l'occurrence, les molécules indicatrices ressortissent à la classe des polyphé- 
nols, groupe des flavonoïdes, largement utilisés dans notre laboratoire. Ces "marqueurs" ne semblent pas avoir été employés jusqu'alors pour le Pin sylvestre, tandis que les terpènes ont été maintes fois sollicités (Juvonen, Hiltunen, 1972, 1974 ; Hiltunen, 1975, 1976 ; Hiltunen et al, 1975a et b ; Lange, Weissmann, 1988 ; Tigerstedt et al, 1978 ; Yazdani et al, 1982, 1985, 1986) de même que les isoenzymes (Rudin, 1975, 1976, 1977 ; Rudin, Ekberg, 1978), sans pour autant résoudre totalement, semble-t-il, les problèmes systématiques posés.

Le présent travail est le développement d'une thèse de $3^{e}$ cycle (Laracine, 1984) ; des notes préliminaires ont été publiées (Lebreton, Laracine, 1984 ; Laracine, Lebreton, 1985 ; Laracine-Pittet, Lebreton, 1988). II correspond au plan de la biochimie foliaire à l'interrogation soulevée par Birot et Lacaze (1981) quant à la provenance des graines utilisées en reboisement, et prétend fournir des éléments de jugement pour une meilleure caractérisation théorique et pratique du complexe spécifique Pinus sylvestris.

\section{MATÉRIEL ET MÉTHODES}

Echantillonnage (au niveau des populations) et analyse (au niveau des individus) doivent être particulièrement soignés pour de telles études.

\section{Échantillonnage botanique}

Les populations $n^{\text {os }} 4$ à 10 (tableau 1) et les pins d'Ecosse fournis par MM Forrest et Phillips ont été prélevés in natura dans des conditions garantissant leur indigénat. Les échantillons de Suede (Dr Yazdani) et ceux provenant de l'INRA (basses Vosges, reste de la France, pays de l'Est) sont issus de plantations comparatives (Amance, Royat, les Barres ou Bout) (Lacaze, 1964) (tableau II).

La thèse précitée a permis de montrer que :
- l'expression flavonique est bien déterminée génétiquement, la variabilité intraclonale (éprouvée sur 10 clones différents, à raison de 4 plants greffés par clone) étant du même ordre de grandeur ( \pm 7 à $15 \%$ selon les molécules, en teneur relative) que la variabilite intra-individuelle :

- exception faite des jeunes aiguilles (de juin à août) de l'année, le contenu flavonique ne subit pas de variations saisonnieres et interannuelles importantes ( $\pm 7-9 \%$ sur 12 mois consécutifs chez le même individu, pour 3 marqueurs affectés d'une incertitude strictement analytique égale à $2.4 \%$ ), variations bien inférieures à la plupart des différences notées entre individus distincts :

- enfin, les mémes clones cultivés dans des arboreturns différents (et distincts de la station naturelle d'origine) montrent des teneurs flavoniques tout à fait comparables, les écarts étant là encore du meme ordre de grandeur que ceux notés dans le même site entre greffons issus du même clone, ou entre échantillons prélevés sur le même individu.

Les figures 1 et 2 illustrent clairement l'ampleur de cette variabilité qui, globalement estimé à $\pm 10 \%$ en pratique, permet de formuler toutes conclusions face à des différences parfois supérieures à $100 \%$ entre individus distincts, souvent issus de la même population.

\section{Analyse biochimique}

L'analyse a porté sur des aiguilles aduites séchees à l'air libre, à l'abri de la chaleur et de la lumière vives. Exposé en détail par ailleurs (Laracine, 1984), le mode operatoire est basé sur le traitement acide $(\mathrm{HCl} 2 \mathrm{~N}$, $160 \mathrm{ml}$ aq pour $2 \mathrm{~g}$ de poudre végétale ; $45 \mathrm{~min}$ au bain-marie bouillant) des proanthocyanidines (qui génèrent les anthocyanidines homologues, cyanidine et delphinidine) et des glycosides flavoniques (qui libèrent les aglycones correspondants : kaempférol, quercétine, isorhamnétine, voire taxifoline (= dihydro-2,3 quercétine)) .

\footnotetext{
Lundgren et Theander (1988) ont récemment confirmé la présence de taxifoline dans les aiguilles du Pin sylvestre, et précisé sa structure sous forme glucosidique; ils signalent également l'existence de chimiotypes basés sur ce flavonoïde.
} 


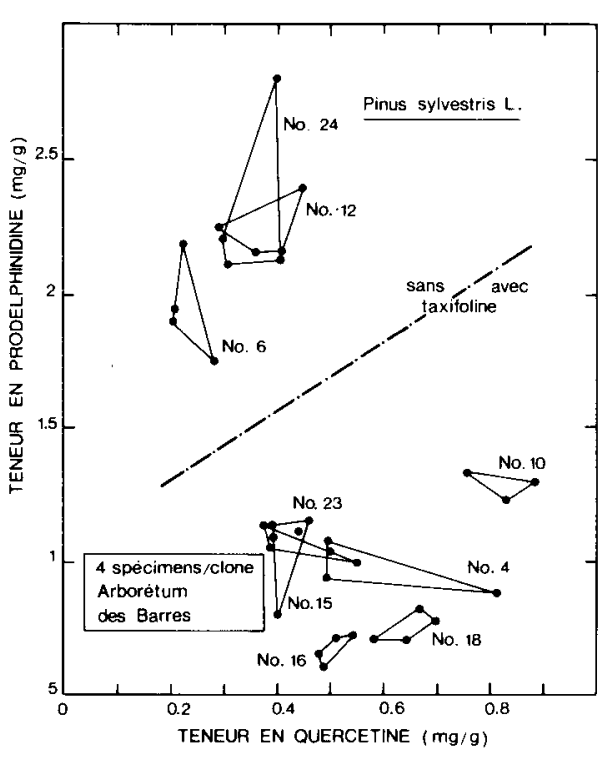

Fig 1. Constance intraclonale des caractères flavoniques.

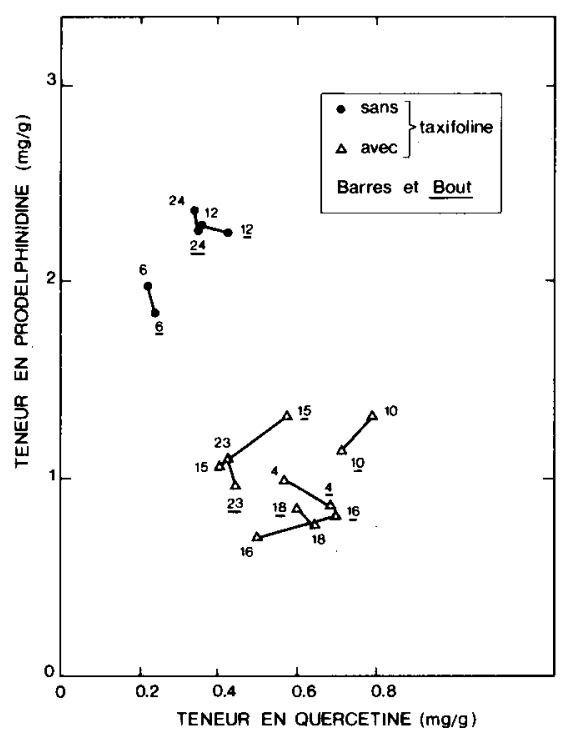

Fig 2. Variabilité des caractères flavoniques en fonction du lieu de culture (moyennes clonales).
Les anthocyanidines totales sont dosées en phase aqueuse à $535 \mathrm{~nm}$, les flavonols à $425 \mathrm{~nm}$ après extraction à l'éther, reprise par l'éthanol et complexation aluminique. Les proportions des substances sont déterminées par CLHP (chromatographie liquide haute performance ; colonne C18 MicroBondapak ; solvant $\mathrm{MeOH} / \mathrm{H} 20 / \mathrm{AcOH}$ : $30 / 60 / 10$ pour les anthocyanidines, 40/55/05 pour les flavonols). Le produit de la teneur globale en flavonoides par la teneur relative (mesurée en hauteur de pics) donne la teneur absolue $\left(\mathrm{mg}^{-1} \mathrm{~g}^{-1}\right)$ de chaque composé. Bien que la taxifoline apparaisse - avant les flavonols - en CLHP, il est plus commode de la détecter par CCM (chromatographie sur couche mince ; polyamide Merck $11 \mathrm{~F}$ 254 ; solvant $\mathrm{Bz} / \mathrm{MeOH} / \mathrm{MEC}: 40 / 30 / 40$, Rf 0,57 , fluorescence brune) (fig 3 pour des profils CLHP).

\section{RÉSULTATS ET DISCUSSION}

\section{Reconnaissance d'un polymorphisme flavonique}

Une premiere population (Hanau, basses Vosges du Nord; 30 spécimens prélevés dans des plantations INRA) a été étudiée de manière approfondie. Le fait marquant est la coexistence de 2 chimiotypes bien distincts (fig 4) : le premier (17 spécimens) est marqué par la prédominance de la prodelphinidine, en valeurs relatives $(90 \pm 2 \%$ du total des 2 proanthocyanidines présentes) comme absolues $\left(2,03 \pm 0,29 \mathrm{mg} \cdot \mathrm{g}^{-1}\right)$; le second (13 spécimens) voit la phényl-trihydroxylation régresser au profit de la dihydroxylation. En effet, non seulement la prodelphinidine est alors concurrencée par la procyanidine (prodelphinidine : $56 \pm 5 \%$ et $0,95 \mathrm{mg}^{-1}$, alors que la procyanidine, précédemment égale à $0,23 \mathrm{mg}^{-1} \mathrm{~g}^{-1}$ vient ici à $0,75 \mathrm{mg}^{-1} \mathrm{~g}^{-1}$, mais la quercétine double en valeur absolue (elle passe de 0,29 $\pm 0,08 \mathrm{mg} \cdot \mathrm{g}^{-1}$ à $0,62 \pm 0,14 \mathrm{mg} \cdot \mathrm{g}^{-1}$; de $23 \pm 5$ à $30 \pm 4 \%$ du total des fla- 

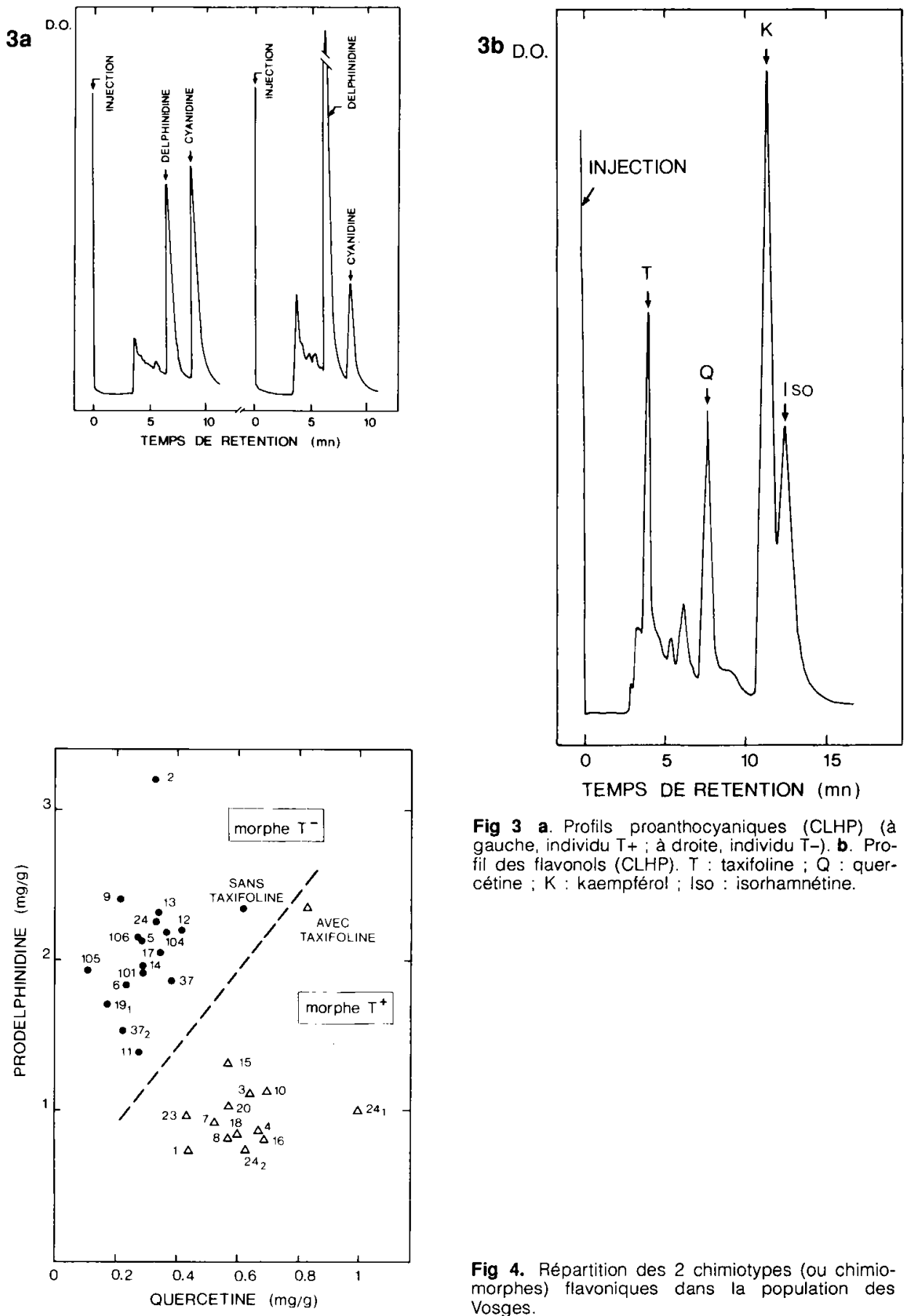

Fig 3 a. Profils proanthocyaniques (CLHP) (à gauche, individu $\mathrm{T}+;$ à droite, individu $\mathrm{T}-$ ). b. Profil des flavonols (CLHP). T : taxifoline ; $Q$ : quercétine : $\mathrm{K}$ : kaemptérol ; Iso : isorhamnétine.

Fig 4. Répartition des 2 chimiotypes (ou chimiomorphes) flavoniques dans la population des vosges. 


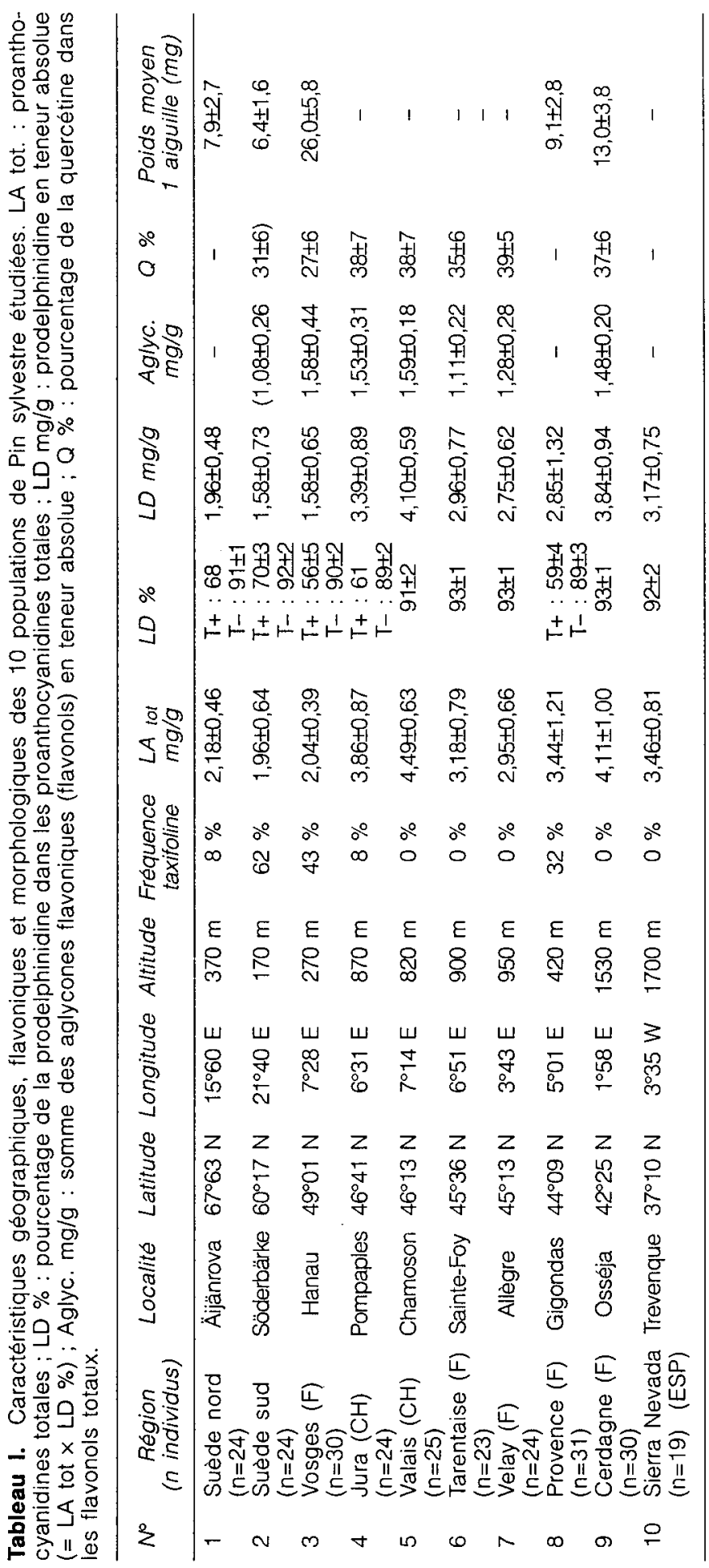


Tableau II. Composition flavonique de diverses origines européennes de Pin sylvestre, cultivés en arboretums (INRA, SUAS) ou prélevés in natura (autres origines). Mêmes symboles que pour le tableau 1. Entre parenthèses: écarts types.

\begin{tabular}{|c|c|c|c|c|c|c|c|c|c|}
\hline \multirow{2}{*}{$\frac{N^{p}}{11}$} & \multirow{2}{*}{$\begin{array}{l}\text { Origine } \\
\text { Ecosse }\end{array}$} & \multicolumn{2}{|c|}{ Echantillons } & \multicolumn{2}{|c|}{$\begin{array}{c}\text { Frequence } \\
\text { taxifoline }\end{array}$} & \multirow{3}{*}{$\begin{array}{l}\text { LD \% } \\
95(4) \\
87(3) \\
88(4)\end{array}$} & \multirow{3}{*}{$\begin{array}{l}\angle D \mathrm{mg} / \mathrm{g} \\
2,61(0,81) \\
2,84(0,60) \\
2,02(0,61)\end{array}$} & \multirow{3}{*}{ 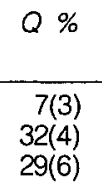 } & \multirow{3}{*}{$\begin{array}{c}Q m g / g \\
0,06(0,02) \\
0,43(0,05) \\
0,43(0,10)\end{array}$} \\
\hline & & $\begin{array}{r}12: \\
4:\end{array}$ & $\begin{array}{l}\text { Ecos } \\
\text { INR }\end{array}$ & $\begin{array}{l}0 \% \\
0 \%\end{array}$ & & & & & \\
\hline 12 & $\begin{array}{l}\text { Pays Est } \\
\text { (URSS, Pol, }\end{array}$ & $23:$ & & & $15 T-$ & & & & \\
\hline & $\begin{array}{l}\text { Bulg, Roum, } \\
\text { Tchécosl) }\end{array}$ & & & & $8 \mathrm{~T}+$ & $50(7)$ & $0,72(0,11)$ & $35(3)$ & $0,72(0,15)$ \\
\hline \multirow[t]{8}{*}{13} & Scanc & & & & $7 T-$ & $90(2)$ & $2,48(1,12)$ & $29(5)$ & $0,47(0,12)$ \\
\hline & & 8 & & $\circ$ & $1 T_{1}$ & & & 32 & 0,19 \\
\hline & Suède nord & 10: & SUAS & $0 \%$ & & $\begin{array}{l}90(2) \\
92(2)\end{array}$ & & - & - \\
\hline & Suede centre & $19:$ & SUAS & $26 \%$ & & & & & \\
\hline & & & & & & $65(6)$ & & - & - \\
\hline & Suède sud & 9: & SUAS & $33 \%$ & & $93(1)$ & & - & - \\
\hline & & & & & $3 T+$ & $59(4)$ & 0,5 & - & - \\
\hline & $\begin{array}{c}\text { Finlande } \\
\text { centre }\end{array}$ & 7: & SUAS & $0 \%$ & & $93(1)$ & & - & - \\
\hline \multirow[t]{2}{*}{14} & $\begin{array}{l}\text { Alpes } \\
\text { France }\end{array}$ & 13: & INRA & $15 \%$ & $11 \mathrm{~T}-$ & $90(1)$ & 2,2 & $31(4)$ & 0,4 \\
\hline & Suiss & 9 : & $\begin{array}{l}\text { unive } \\
\text { Laus }\end{array}$ & $0 \%$ & $2 \mathrm{~T}+$ & $\begin{array}{l}55-66 \\
91(1)\end{array}$ & $\begin{array}{l}0,95 \\
3,41(0,62)\end{array}$ & $\begin{array}{l}27-30 \\
33(3)\end{array}$ & $\begin{array}{l}0,79(0,05) \\
0,55(0,08)\end{array}$ \\
\hline \multirow[t]{3}{*}{15} & $\begin{array}{l}\text { Massif Central } \\
\text { France }\end{array}$ & $30:$ & INRA & $7 \%$ & $28 \mathrm{~T}-$ & $89(2)$ & $2,37(0$ & $27(3)$ & $0,39(0,10)$ \\
\hline & & & & & $\begin{aligned} 2 \mathrm{~T}+ \\
11 \mathrm{~T}-\end{aligned}$ & $\begin{array}{l}50-55 \\
95(1)\end{array}$ & $\begin{array}{l}1,00 \\
3,25(0,90)\end{array}$ & $\begin{array}{c}35-37 \\
-\end{array}$ & $\begin{array}{c}0,76(0,09) \\
-\end{array}$ \\
\hline & $\begin{array}{l}\text { France } \\
\text { Pyrenées }\end{array}$ & $12:$ & $\begin{array}{l}\text { université } \\
\text { Clermont-Fd }\end{array}$ & $8 \%$ & $1 \mathrm{~T}+$ & 73 & 1,34 & - & - \\
\hline \multirow{2}{*}{16} & France & 8 & INRA & $0 \%$ & & $90(2)$ & $2,97(0,36)$ & $26(3)$ & $0,33(0,08)$ \\
\hline & & & 142 & 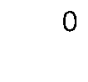 & $\%$ & 87 à 95 & 1,15 & $\begin{array}{l}(7) 26 \\
\text { à } 32\end{array}$ & $\begin{array}{l}(0,06) 0,33 \\
\text { à } 0,49\end{array}$ \\
\hline \multicolumn{2}{|c|}{164 échantillons } & & 2 & & & 50 à 73 & 0,52 à 1,59 & $\begin{array}{c}(27) 30 \\
\text { à } 37 \\
\end{array}$ & $\begin{array}{r}0,72 \\
\text { a } 0,79 \\
\end{array}$ \\
\hline
\end{tabular}

vonols) (toutes ces différences sont hautement significatives au test $t$ de Student). En outre, la taxifoline apparaît, alors qu'elle était absente du premier chimiotype.

La corrélation de ces divers caractères relève d'une évidente logique biochimique (fig 5). On peut même penser que la détection des 4 formes flavoniques permet en fait d'éprouver 3 sys- tèmes enzymatiques soumis à variabilité génétique : une ortho-phényl- hydroxylase antagoniste de 2 (alcool-)déshydrogénases corrélées. Le caractère "taxifoline" étant le plus tranché, et le plus commode à détecter en routine, nous le retiendrons pour qualifier couramment les 2 chimiotypes, dits $\mathrm{T}$ - et $\mathrm{T}+$ (taxifoline absente et présente, respectivement). Le second chimiotype est 


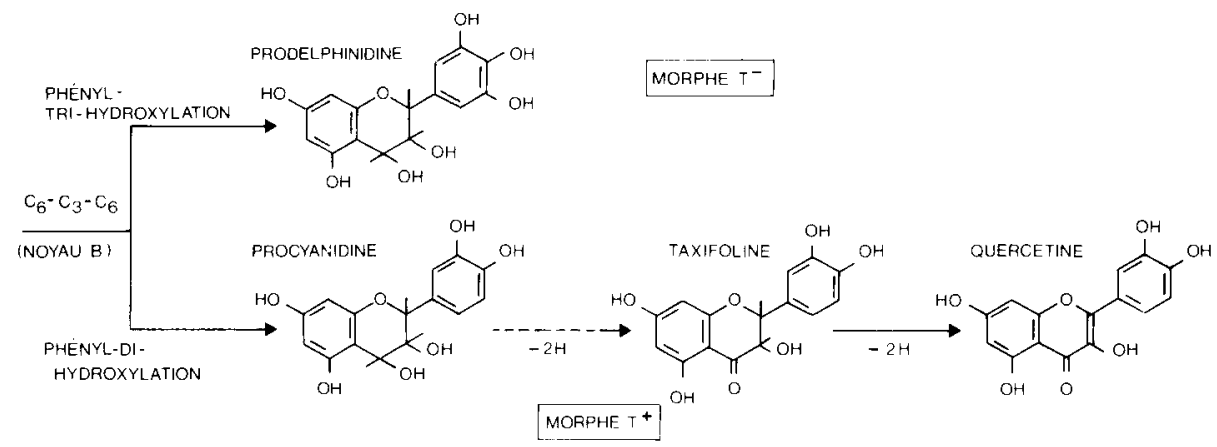

Fig 5. Relations biosynthétiques des flavonoïdes.

plus diversifié et plus oxydé, donc plus "évolué", le premier plus "primitif", caractère d'ailleurs classiquement reconnu à la prodelphinidine (fig 6).

Plus exactement, nous sommes ici en présence d'un phénomène de polymorphisme (de dimorphisme) au sens de Ford, et les 2 chimiotypes peuvent être considérés également comme 2 «morphes", chimiomorphes plus précisément. On notera que ce caractère biochimique n'est pas corrélé, par exemple, au poids des aiguilles : 27,8 $\pm 5,7 \mathrm{mg}$ pour les individus relevant du premier morphe, $24,1 \pm 5,9 \mathrm{mg}$ pour les seconds.

\section{Aspects biogéographiques du polymorphisme flavonique}

De même qu'au sein de la précédente population, la situation est hétérogène au niveau biogéographique européen : si l'on rencontre (tableau I) des populations où la fréquence des 2 chimiotypes est du même ordre de grandeur que dans les Vosges (Suède sud : 15 individus T+ sur 24 ; Provence : 10 in-

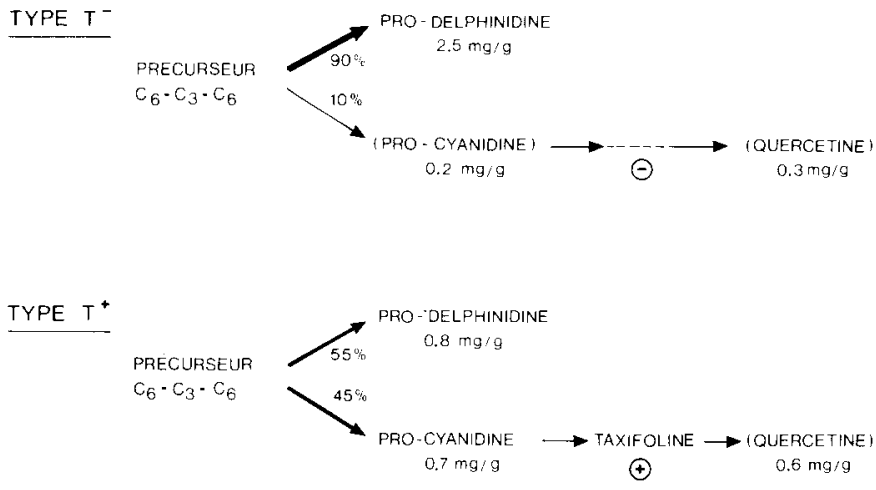

Fig 6. Caractéristiques des 2 types (ou morphes) flavoniques du Pin sylvestre. 
dividus $T+$ sur 31 ), 7 autres populations ne connaissent pratiquement que le morphe T-: Alpes (Valais, $\mathrm{CH}$; Tarentaise, F), Massif central (Velay), Pyrénées (Cerdagne, F), Sierra Nevada (ESP) : $0 \%$; Suede (nord du cercle polaire) et Jura $(\mathrm{CH}): 8 \%$.

A ce tableau, on peut ajouter : 28 autres individus faisant passer à $44 \%$ l'estimation de la fréquence générale du morphe $T+$ en Suède centrale et méridionale; 23 individus (collections INRA) en provenance de l'Est (Russie, Tchécoslovaquie, Pologne, etc), où le chimiotype $\mathrm{T}+$ connaît une fréquence de $35 \%$; 12 individus provenant d'Ecosse, non seulement tous $T-$ mais avec une teneur très basse en quercétine ${ }^{* *}: 7 \pm 3 \%$ du total des flavonols et $0,06 \pm 0,02 \% \mathrm{mg} \cdot \mathrm{g}^{-1}$ en valeur absolve, tandis que la prodelphinidine monte à $98 \pm 1 \%$ du total des proanthocyanidines ${ }^{\star \star \star}$ (tableau $\|$ et fig 7 ).

\section{Déterminisme du polymorphisme flavonique}

La distribution des 10 populations étudiées traduit une dépendance étroite de la fréquence du morphe $\mathrm{T}+$ envers l'altitude et/ou la latitude (fig 8). Les 7 populations où le chimiotype $T-$ se trouve fixé sont en effet de cote $(>800 \mathrm{~m})$ ou de latitude $\left(>67^{\circ} \mathrm{N}\right)$ éle-

\footnotetext{
Soit en pourcentage : 43,62 et $32 \%$ respectivement. Si Suède-sud et Provence diffèrent $\left(\chi^{2}\right.$, $p<0,05$ ), ni l'un ni l'autre ne se distingue des Vosges. En outre, aucune des 3 populations ne diffère significativement d'une distribution $T+/ T$ $50 / 50$.

Toutefois, 4 individus écossais des collections INRA ne se distinguent pas des échantillons Tcontinentaux (prodelphinidine : $86 \pm 3 \%$; quercétine : $33 \pm 4 \%$ et $0,43 \pm 0,06 \mathrm{mg}^{-1}$ ).

". On constate également que chez les individus $T+$, la teneur en prodelohinidine est plus forte au nord (Suede : 68 et $70 \pm 3 \%$ ) qu'au sud (Vosges : $56 \pm 5 \%$; Provence : $59 \pm 4 \%$ ), ce qui traduit peut-être une certaine introgression Tchez les premiers.
}

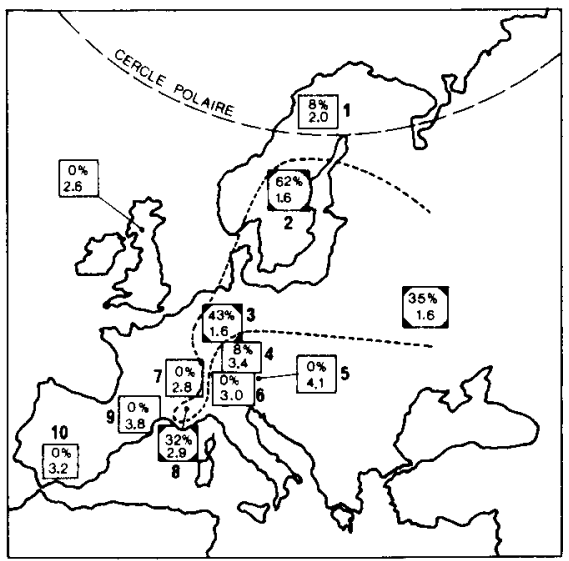

Fig 7. Répartition géographique des 2 types flavoniques "A" et "P" du $P$ in sylvestre.

P. : type de plaine (taxifoline présente) ;

A. : type d'altitude (taxifoline absente, ou rare) $(32 \%=$ fréquence $\mathrm{T}+; 2,9=\mathrm{LD} \mathrm{mg} / \mathrm{g})$.

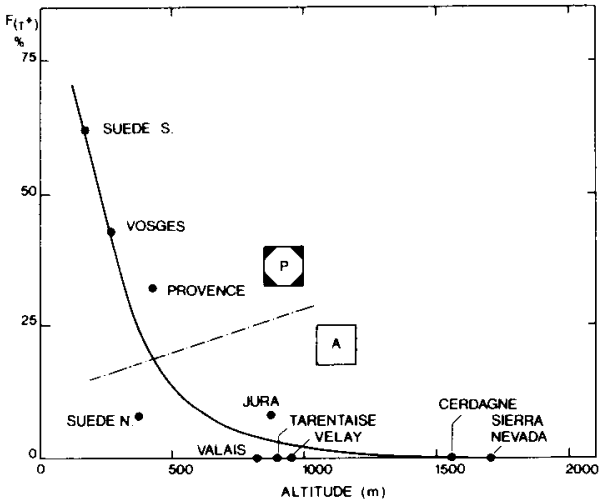

Fig 8. Déterminisme altitudinal du polymorphisme flavonique.

vées, contrairement aux 3 autres ; il existe en outre une corrélation hautement significative entre l'altitude de la station d'origine et la teneur absolue en 
prodelphinidine chez 48 spécimens français échantillonnés par I'INRA ( $r=$ $+0,688, p<0,001$ ).

Le premier paramètre écologique pouvant être lié à l'altitude est évidemment la température, qui présente en effet une excellente corrélation (négative) avec la teneur en prodelphinidine : $r=-0,628(p<0,001)$ pour la moyenne annuelle " la liaison - toujours hautement significative - est meilleure avec la température moyenne de juin ( $r=$ - 0,682) qu'avec celle de janvier ( $r=$ - 0,472). Une relation significative existe, mais moins forte, avec l'insolation annuelle $(r=+0,413)$, mais elle est nulle avec la pluviosite annuelle ( $r=$ $+0,079$ ) (données climatiques tirées de Arlery, 1979). En d'autres termes, le chimiotype $T+$ peut être considéré comme thermo(hélio)phile, et le chimiotype T- (biochimiquement plus économe : y a-t-il relation causale ?) qualifierait les populations et individus "froids" de l'espèce Pinus syivestris.

Cependant, le cas de la population de Sierra Nevada $\left(1700 \mathrm{~m}\right.$; $\mathrm{T}_{+}=$ $0 \%$; $L D=3,2 \mathrm{mg} \cdot \mathrm{g}^{-1}$ ) suffirait à lui seul à dénier une soumission aussi directe du dimorphisme $T+/ T-$ à un déterminisme thermique actuel : bien qu'élevée, cette population franchement méditerranéenne se trouve en effet en situation chaude (à l'étage du chêne vert $Q$ ilex ). II convient donc plutôt de placer le problème dans un contexte historique et évolutif : dans l'étage montagnard (et, d'une manière générale, au-dessus de $1000 \mathrm{~m}$ ), l'aire actuelle du Pin sylvestre (Alpes, Jura, Massif central, Pyrénées, Sierra Nevada) correspond sans doute à la ré-extension vers le bas des stations-refuges

\footnotetext{
Voir aussi la figure 9 pour un ajustement curviligne des points expérimentaux; la même courbe est obtenue pour des arbres situés dans 2 arboretums différents.
}

d'altitude occupées lors des maximums thermiques interglaciaires; $i$ en est de même au-delà du cercle polaire.

On peut ainsi voir dans le chimiotype T- la souche "boréo-alpine» de l'espèce, et penser qu'il constituait (en Europe moyenne) la seule forme lors des épisodes glaciaires. Entre les crises froides et chaudes (nous sommes actuellement en phase de transition), la plaine a pu offrir le champ libre à la (re)conquête (depuis l'est, ou le sud ?) par le morphe thermophile $T+$, qui représente actuellement un à deux tiers des individus, en équilibre avec le morphe "fondamental» $T-$. Le hiatus biogéographique entre nord et sud de l'Espagne n'a pas autorisé une telle reconquête, comme en France ou plus au nord-est, d'où la présence exclusive en Sierra Nevada du type T- en situation chaude, hors de toute concurrence chimiomorphique. L'étude de la transmission génétique des caractères T+ et T- (Yazdani et Lebreton, à parâ̂tre) pourra expliquer les modalités de ces phénomènes.

\section{Propositions systématiques}

Bien qu'un seul caractère (chimique ou non) ne puisse généralement suffire à fonder une classification, la mise en évidence du dimorphisme $T+/ T$ - et de son extension peut éclairer d'un jour nouveau les "systématiques" jusqu'alors proposées pour le Pin sylvestre.

Le faible taux en quercétine apporte certes un argument supplémentaire à la reconnaissance d'un taxon (variété ou sous-espèce) scotica autonome. Mais les diverses fréquences du chimiotype $T+$, ainsi que la teneur en prodelphinidine, ne justifient pas l'individualisation des nombreuses variétés reconnues çà et là : comment distin- 
guer ici la var nevadensis Christ des autres taxons d'altitude, ou bien encore les var rigensis Loud, ou bororussica Schott du "pin des Vosges" var haguenensis Loud (in Gaussen, 1960) ?

L'examen de la carte flavonique obtenue (fig 7) suggère une position plus prudente, consistant à reconnaître (hors scotica Schott ?) 2 sous-ensembles "A" (comme altitude) et “P» (comme plaine), caractérisés non seulement par la fréquence de la taxifoline, mais par la teneur - corrélativement plus basse - de la prodelphinidine : en moyenne $1,9 \pm 0,6 \mathrm{mg} \cdot \mathrm{g}^{-1}$ pour $P$ contre $3,1 \pm 0,7 \mathrm{mg} \cdot \mathrm{g}^{-1}$ pour $\mathrm{A}$ (différence statistiquement significative : $t=$ 2,$65 ; \mathrm{ddl}=10 ; p=0,03$ ).

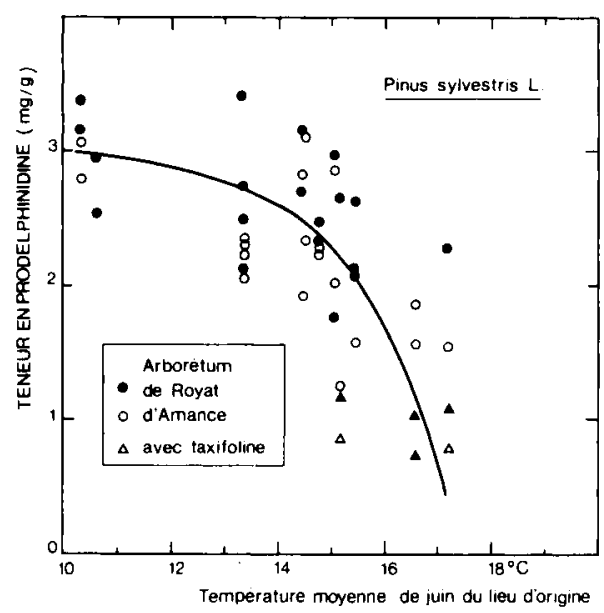

Fig 9. Déterminisme thermique du polymorphisme flavonique.

En fait, cette proposition n'est pas aussi originale qu'on pourrait le penser ; dans son traité de 1960, Gaussen n'écrivait-il pas: “M. Guinier émet une idée qui paraît très séduisante : les races «nobles» du Nord auraient pro- gressé vers l'Europe centrale lors des périodes glaciaires et auraient donné les belles races montagnardes" ( $=$ notre type $A, T-$ ). "Par contre, lors des interglaciaires, des Pins méridionaux ou d'Europe centrale de forme défectueuse " auraient progressé vers le Nord. Ils seraient restés au bas des montagnes» ( $=$ notre type $\mathrm{P}, \mathrm{T}+$ ) «et pourraient avoir une origine orientale".

Nous retrouvons peut-être aussi le point de vue synthétique et volontairement simplificateur adopté par Probst (1983) à l'issue d'une revue taxonomique de l'espèce dont elle retire que “Mirov (1967) et De Ferre (1979) sont d'accord pour conclure à l'existence de 2 Pins sylvestres : un septentrional et un méridional». Dans cette hypothèse, les "groupes" définis par Gaussen pourraient se répartir comme suit :

- type A, à chimiomorphe T- largement prédominant :

- groupe 1: lapponica Fries (nord de la Suède) ;

- groupe III : scotica Schott ;

- groupe IV : “montagnards» dont aquitana Schott d'Auvergne, et pyrenaica Svob;

- type $P$, à chimiomorphes $T+$ et $T-$ coprésents :

- groupe II : septentrionalis Schott (centre et sud de la Suède);

- groupe V : «basses altitudes de l'aire moyenne et méridionale", dont le var haquenensis Loud, mais non les var engadinensis Herr et nevadensis Christ.

D'un point de vue pratique, la clé chimiotaxinomique ici proposée présente éventuellement un triple intérêt :

Encore que rien de tel n'apparaisse dans nos échantillons : bien au contraire, les pins des Vosges ici étudiés étaient de bonne forme au sens des forestiers. 
- elle peut permettre d'éprouver a posteriori l'origine, autochtone ou non, de boisements pour lesquels les archives feraient défaut. Ainsi, l'indigénat d'un peuplement situé à $500 \mathrm{~m}$ d'altitude sera douteux si aucun individu $T+$ n'y peut être décelé ;

- elle peut guider l'échantillonnage pour toute étude biologique à conduire sur l'espèce. Des corrélations physiologiques sont d'ailleurs possibles, les flavonoïdes paraissant tenir un rôle d'indicateurs à l'égard du “dépérissement forestier" chez les coniferes (cf Louguet in Queiroz, 1987) ;

- elle peut aider le forestier dans sa politique de reboisement, complétant d'autres critères plus classiques. Le praticien évitera ainsi d'implanter, ou de maintenir, des individus $\mathrm{T}+$ en altitude, et les favorisera plutôt pour toute operation conduite dans l'étage collinéen.

\section{Corrélations biochimiques}

De nombreuses études ont été consacrées (vide supra) à la composition terpénique des "résineux" en général, du Pin sylvestre en particulier, dans l'espoir de contribuer à une meilleure définition des taxons. Il était donc tentant de voir si, d'une part les spécialistes respectifs des terpènes et des polyphénols aboutissaient aux mêmes propositions et si, d'autre part, des corrélations existaient entre ces 2 familles moléculaires.

Dans un récent article, Ohalchat et al (1985) ont rapporté la découverte, dans le Massif central, de 2 populations de Pin sylvestre aux profils terpéniques (déterminés sur branchettes) totalement différents : la première population (col de la Moréno, altitude
$1000 \mathrm{~m}$; Puy-de-Dôme) possède du $\Delta-3$ carène à la teneur relative de 32 $\pm 10 \%$ dans le total des monoterpènes ; la seconde (Cissac-Saint-11pize, altitude $600 \mathrm{~m}$, Haute-Loire) ignore pratiquement ce constituant, au profit (relatif) de l' $\alpha$ et du $\beta$-pinène.

L'analyse terpénique (conduite sur les aiguilles de $6+6$ individus fournis par le professeur Garry, de l'université de Clermont-Ferrand) donne des résultats très homogènes pour Saint-llpize $\left(\mathrm{LA}\right.$ (tot) $=3,36 \pm 0,33 \mathrm{mg} \cdot \mathrm{g}^{-1}$; Flav (tot) $=0,50 \pm 0,07 \mathrm{mg}^{-1} ; \mathrm{T}_{+}=0 \%$ ), alors que la population de la Moréno est polymorphe ( $L A=2,83 \pm 1,42$ mg.g- $^{1}$; Flav $=0,72 \pm 0,17 \mathrm{mg} \cdot \mathrm{g}^{-1} ; \mathrm{T}+=17 \%$, \pm 1 individu sur 6 ). Ces données ne sont pas globalement contradictoires avec nos précédentes analyses ( $c f$ tableau I: Velay ; tableau II : Massif central, INRA), mais portent sur un échantillon trop restreint pour autoriser des conclusions définitives. On constate néanmoins que les "caractères" taxifoline et $\Delta-3$ carène ne sont pas obligatoirement liés, comme le sont entre eux les caractères taxifoline et prodelphinidine, par exemple.

De même, 2 autres populations, suédoises celles-ci (cf tableau I; Suède nord et sud), ont-elles été étudiées en ce qui concerne a la fois les polyphénols et les terpènes (Yazdani et al, 1985). Bien distinctes quant à la fréquence du chimiotype $T+$ (8 et $62 \%$ respectivement), elles ne semblent pas très éloignées du point de vue terpénique : leurs teneurs en $\Delta-3$ carène sont élevées, et identiques $(40,2 \pm 0,5$ vs $39,6 \pm 0,6 \%$ ), et si le rapport $\alpha$-pinène/ $\beta$-pinène l'emporte chez la population méridionale (il est égal à 1,65 contre 0,60 au nord), c'est avec des valeurs bien différentes de celles notées dans le Massif central (6,0 à La Moreno contre 2,2 à Saint-llpize). 
Sous réserve d'inventaire, métabolismes terpénique et polyphénolique semblent donc évoluer indépendamment, ce qui ne surprend pas outre mesure à la réflexion, puisque l'un dépend de la voie du mévalonate, l'autre de la voie du shikimate, très différentes du point de vue de la biogenèse chimique ; ils ne peuvent donc vraisemblablement pas se prêter aux mêmes exploitations taxonomiques, mais néanmoins se compléter pour des calculs de distances génétiques basés sur un plus grand nombre de constituants.

En fait, le marqueur terpénique semble plus utile à moyenne échelle : Yazdani et al (1985) ont ainsi montré l'existence d'un cline de la teneur en limonène du nord au sud de la Suede; des conclusions analogues ont été formulées en Finlande (Hiltunen, 1976), notamment pour le $\Delta-3$ carène. Les flavonoïdes, nous l'avons vu, opèrent à une autre échelle, celle d'un continent.

Loin de se concurrencer, ces «images» complémentaires du génome végétal doivent contribuer à une systématique aussi objective et synthétique que possible, permettant ainsi de mieux comprendre, pour mieux la préserver, la diversité du monde vivant, «thème de premier plan qui (devrait réunir) biologistes dits naturalistes et biologistes moléculaires», car il s'agit d'un "facteur essentiel d'adaptation" à propos duquel les "connaissances actuelles apparaissent insuffisantes" (Cauderon, in Arbez, 1988).

\section{REMERCIEMENTS}

Le ministère de la Recherche ( FFilière Bois"), l'Office National des Forêts (MM Martinot-Lagarde et $P$ Legroux) et le ministère de l'Education ("Aide à la Recherche Universitaire") ont financé ce travail. L'INRA (Institut National de la Recherche Agronomique, ministère de l'Agriculture, MM Birot,
Bonnet-Masimbert, Roland et Roman-Amat) a fourni de nombreux echantillons référencés. D'autres échantillons ont été fournis par Mmes Plumettaz-Clot et Neet-Sarqueda (Institut de Botanique systématique et de Géobotanique, université de Lausanne, Suisse), MM Yazdani et Lindgren (département de génétique forestière de physiologie végétale de l'université suédoise des Sciences Agronomiques, Umeá, Suède), M Arpin (laboratoire de mycochimie, université Lyon-I), MM Forrest et Phillips (commission forestiere de Midlothian, Ecosse), M Garry (laboratoire de chimie organique 2, Université Clermont II), Mme Lescourret et $M$ Genard (GEREA, université de Bordeaux-Talence).

\section{RÉFÉRENCES}

Arbez M (1987) Les Ressources génétiques forestières en France. $t$ 1: Les Conifères. INRA et BRG, 169

Arbez M (1988) Méthodes biochimiques de caractérisation variétale des arbres forestiers. Rev For Fr (Nancy) XL, $n^{\circ}$ spécial, 71-76

Arlery R (1979) Le climat de la France. Dir Méteo Nation Editeur, $131 \mathrm{p}$

Birot $Y$, Lacaze JF (1981) Choix des sources de graines de Pin sylvestre. Rev For Fr (Nancy) XXXIII, 187-194

Chalchat JC, Garry RP, Michet A, Remery A (1985) The essential oils of two chemotypes of Pinus sylvestris. Phytochem 24, 2443-2444

De Ferre Y (1979) Apport de l'étude des plantules dans la connaissance du Pin sylvestre. Bull Soc Bot Fr Actual Bot 126, 217-226

Gaussen H (1960) Les Gymnospermes actuelles et fossiles. Fasc VI, Chap XI, Faculté des Sciences de Toulouse Edit, $272 p$

Hiltunen R (1975) Variation and inheritance of some monoterpenes in Pinus silvestris. Planta Med 28, 315-323

Hiltunen R (1976) On variation, inheritance and chemical interrelationships of monoterpenes in Scots Pine (Pinus sylvestris). Ann Acad Sci Fenn 208. In : Biol Abstr (1976) 62, 25822

Hiltunen $R$, Juvonen $S$, Tigerstedt PMA (1975a) Geographical variation in some monoterpenes in Scots Pine (Pinus silves- 
tris ) in Finland. Farm Aikak 84, 73-82. In : Chem Abstr (1976) 84, $28136 \mathrm{~g}$

Hiltunen R, Tigerstedt PMA, Juvonen S, Pohjola $J$ (1975b) Inheritance of 3-carene quantity in Pinus silvestris. Farm Aikak 84, 69-72. In : Chem Abstr (1976) 84, $40847 \times$

Jalas J, Suominen J (1973) Distribution of vascular plants in Europe. 2. Gymnospermae. Atlas Florae Eur 28-31

Juvonen S, Hiltunen R (1972) Occurrence of 3-carene poor and 3-carene rich chemotypes in Pinus silvestris in Finland. Biogenetic studies. Farm Aikak 81, 137-145. In : Chem Abstr (1973) 78, 145202 n

Junoven S, Hiltunen R (1974) New knowledge of the terpene biosynthesis in Pinus sylvestris. Biogenetic studies through data treatment. Farm Aikak 83, 71-82, In: Chem Abstr (1975) 82, $70291 \mathrm{n}$

Lacaze JF (1964) Comparaison de diverses provenances de Pin sylvestre (Pinus sylvestris L) représentées dans les arboretums forestiers. Rev For Fr (Nancy) XVII, 658-686

Lange W, Weissmann G (1988) Die Zusammensetzung der Harzbalsame von Pinus sylvestris $L$ verschiedener Herkünfte. Holz Roh-Werkstoff 46, 157-161

Laracine C (1984) Étude de la variabilité flavonique infraspécifique chez visux Conifères : le Pin sylvestre et le Genévrier commun. Thèse doctorat $3^{e}$ cycle, université Lyon-I, $n^{\circ} 1424,193 p$

Laracine C, Lebreton P (1985) Le Pin sylvestre, une espèce flavoniquement polymorphe. $7^{\mathrm{e}}$ réunion. Gr Fr Genet Biol Pop, Montpellier

Laracine-Pittet C, Lebreton $P$ (1988) Flavonoid variability within Pinus sylvestris. Phytochem 27, 2663-2666

Lebreton P, Laracine C (1984) Les proanthocyanes, marqueurs infraspécifiques chez des Conifères. CR Coll Gr Polyphénols, Plovdiv 12, 168

Lundgren LN, Theander $O$ (1988) Cis- and Trans- Dihydroquercetin glucosides from needles of Pinus sylvestris. Phytochemistry $27,829-832$
Probst A (1983) Variation intraspécifique et introgression entre Pinus uncinata Ram et Pinus sylvestris $L$ en forêt d'Osséja (Pyrénées-Orientales). Thèse doctorat $3^{e}$ cycle, université de Toulouse-1II, $221 \mathrm{p}$

Queiroz O (1987) Caractérisation de l'activité physiologique d'épicéas soumis à pollution contrôlée et d'épicéas sains ou dépérissants en forêt. In : Les Recherches en France sur le dépérissement des forêts. Programme DEFORPA. EGREF, 61, $88 \mathrm{p}$

Rudin D (1975) Inheritance of glutamateoxalate-transaminase (GOT) from needles and endosperms of Pinus sylvestris. Hereditas 80, 296-300. In : Chem Abstr (1976) $84,56626 \mathrm{k}$

Rudin D (1976) Biochemical genetics and selection application of isozymes in tree breeding. In : IUFRO Joint Meeting on Advanced Generation Breeding, Bordeaux. INRA

Rudin D (1977) Leucine amino-peptidase (LAP) from needles and macrogametophytes of Pinus sylvestris $L$ inheritance of allozymes. Hereditas 85, 219-225

Rudin D, Ekberg I (1978) Linkage studies in Pinus silvestris using macro-gametophytes allozymes. Silvae Genet 27, 112

Tigerstedt PMA, Hiltunen R, Chung MS, Moren E (1978) Inheritance and genetic variation of monoterpenes in Scots Pine (Pinus sylvestris). In : Proc Cont Biochem Genet Forest Trees, Umea. D Rudin Edit

Yazdani R, Nilsson JE (1986) Cortical monoterpene variation in natural populations of Pinus sylvestris in Sweden. Scand J For Res 1, 85-93

Yazdani $R$, Rudin D, Alden T, Lindgren $D$, Harbom B, Ljung K (1982) Inheritance pattern of five monoterpenes in Scots Pine (Pinus sylvestris L). Hereditas 97. 261-272

Yazdani R, Nilsson JE, Ericsson T (1985) Geographical variation in the relative proportion of monoterpenes in cortical oleoresin of Pinus sylvestris in Sweden. Silvae Genet 34, 201-208 